\title{
Comparison of Various Reconstruction Techniques for Line Projections
}

\author{
G. Kontrym-SznajD ${ }^{a}$, M. Samsel-Czekala ${ }^{a}$ And M. Biasini ${ }^{b}$ \\ ${ }^{a}$ Institute of Low Temperature and Structure Research \\ Polish Academy of Sciences \\ P.O. Box 1410, 50-950 Wrocław 2, Poland \\ ${ }^{b}$ Department of Physics, University of California at Riverside \\ California 92521, USA
}

\begin{abstract}
The most effective and popular algorithms to reconstruct the three-dimensional electron-positron momentum density from the measurements of the two-dimensional angular correlation of annihilation radiation are based on the Fourier transform or polynomial expansion. We compare the efficacy of different methods in reconstructing the momentum density of the rare-earth based compounds ErGa, $\mathrm{CeIn}_{3}$ and model profiles, presenting also our new filtering algorithm.
\end{abstract}

PACS numbers: 71.18.+y, 71.20.Lp, 78.70.Bj, 81.70.Tx

\section{Introduction}

The problem of reconstructing densities from line projections via computerized tomography algorithms has been investigated extensively, mostly for applications in medical diagnostics $[1,2]$. By measuring two-dimensional angular correlation of annihilation radiation (2D ACAR) spectra [3] we also obtain the line integrals of a density but in the extended momentum space $\boldsymbol{p}$ :

$$
g\left(p_{y}^{\prime}, p_{z}^{\prime}\right)=\int_{-\infty}^{\infty} \mathrm{d} p_{x}^{\prime} \rho(\boldsymbol{p})
$$

Three reconstruction techniques based on analytical solutions of Eq. (1) and described in the paper are (i) the Cormack method (CM) [4] modified to include various planar symmetries [5], (ii) the filtered back projections (FBP) [6] and (iii) the modification of the FBP as proposed in Ref. [7]. These algorithms reduce the $3 \mathrm{D}$ reconstruction to multiple reconstructions of $2 \mathrm{D}$ densities, performed independently on adjacent parallel planes, perpendicular to a fixed direction $p_{z}^{\prime}=p_{z}$.

\section{Models and discussion}

i) Generally, 2D functions $g$ and $\rho$ can be expanded into polar Fourier series corresponding to $2 \mathrm{D}$ lattice harmonics. The choice of planes $p_{z}=$ const perpen- 
dicular to the main rotation axis of the crystal, where the number of equivalent directions is maximal, allows one to expand both spectra and densities into the following series $[5]$ :

$$
\begin{aligned}
& \rho(\boldsymbol{p}) \equiv \rho(p, \Theta)=\sum_{n=0}^{\infty} \rho_{n}(p) \cos (n \Theta), \\
& g\left(p_{x}, p_{y}=\mathrm{const}\right) \equiv g(t, \varphi)=\sum_{n=0}^{\infty} N_{n}(t) \cos (n \varphi),
\end{aligned}
$$

with $n=i|R|(i=0,1,2 \ldots)$ where $|R|$ denotes the order of the main rotation axis. In CM the radial parts of projections $g_{l}(r)$ and densities $\rho_{l}(r)$ are given as a series of the Chebyshev of the second kind and the Zernike polynomials, respectively [4].

ii) The FBP technique, proposed by Brooks and Chiro [6], utilizes the convolution filtering and sampling theorem [8] and yields the reconstructed data within two steps

$$
1^{\circ} \quad W\left(s_{i}, \varphi\right)=\frac{g\left(s_{i}, \varphi\right)}{4 \Delta s}-\frac{1}{\pi^{2} \Delta s} \sum_{j=1}^{n} \frac{g\left(s_{j}, \varphi\right)}{(i-j)^{2}},
$$

over all $j$ for which $i-j$ is odd; $\Delta s$ denotes the interval between experimental points.

$$
2^{\circ} \quad \rho(p, \Theta)=\int_{0}^{\pi} W[p \cos (\Theta-\varphi)] \mathrm{d} \varphi .
$$

Since functions $W$ describing electronic densities are strongly varying at the Fermi surface, it is necessary to provide a very fine step $\Delta \varphi$ (even in the case of an isotropic model). Conversely, very strong fluctuations of the reconstructed densities above the Fermi momentum, shown in Fig. 1, affect the resulting $\rho(p, \Theta)$.

iii) In alternative, having a small number of measured projections, one could expand the function $W$ into the same series as in Eq. (2) or (3), getting [7],

$$
\rho(p, \Theta)=\sum_{n} \int_{0}^{\pi} W_{n}[p \cos (\Theta-\varphi)] \cos (n \varphi) \mathrm{d} \varphi .
$$

The modified FBP (MFBP) and CM applied to model densities (i.e. in absence of experimental noise) produce the same results (in the case when $\Delta \varphi=$ $1^{\circ}$ ), unlike the standard FBP that gives strong artificial oscillations — see Fig. 1 where these methods (parts (b) and (c)) are applied to a model spectrum (part (a)).

Figure 2 presents densities reconstructed from four 2D ACAR spectra in $\mathrm{ErGa}_{3}$ [9]. Results have been obtained by applying: (i) CM; (ii) standard FBP with using 4 experimental spectra; (iii) MFBP with large and small $\Delta \varphi\left(11.5^{\circ}\right.$ and $1^{\circ}$, denoted in the figure as MFBP-1 and MFBP-2, respectively). Furthermore, Fig. 2 shows a novel procedure, denoted as lattice harmonics expansion (LHE), applied lately to $\mathrm{LaB}_{6}[10]$. LHE is based on the expansion of densities directly in 3D space via the $3 \mathrm{D}$ lattice harmonics. This method was able to reconstruct in $\mathrm{LaB}_{6}$ 


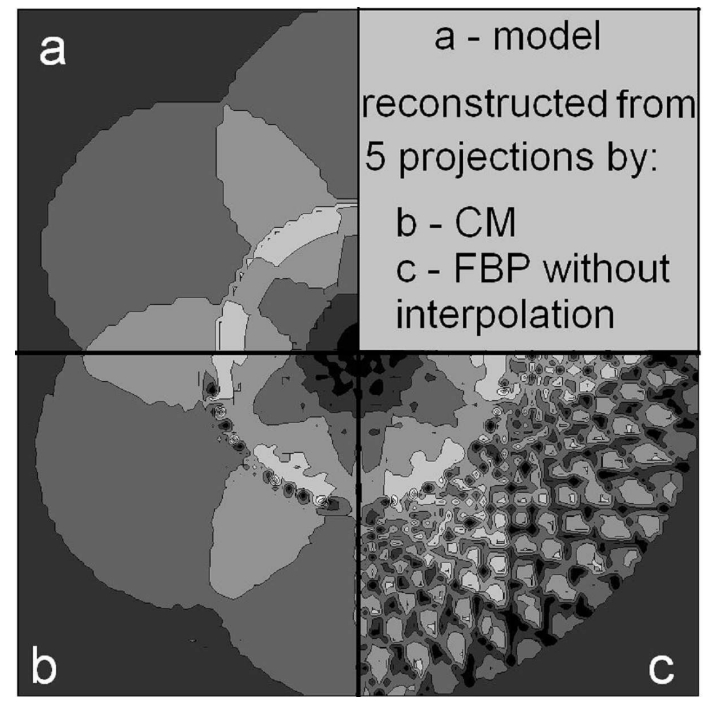

Fig. 1. Model density $\rho(\boldsymbol{p})$ (upper part) reconstructed from five projections (lower part) by applying FBP (c), and CM (b).

a fine piece of the Fermi surface in the 15th band (observed also in the de Haas-van Alphen (dHvA) experiment [11, 12]) that other reconstruction techniques failed to reproduce [13]. Results of the latest band structure calculations in $\mathrm{LaB}_{6}[14]$ (unlike those reported in Refs. $[15,16]$ ) confirmed the existence of this element.

The LHE algorithm, described in detail in Refs. [10, 17], not only filters experimental noise but also enhances details of reconstructed densities since it determines more density components $\rho_{n}(p)$ than the number of spectra used. Indeed, the LHE method, unlike CM, FBP, and MFBP, does not consider the densities on adjacent reconstructed planes, normal to the rotation axis, as independent. Quite the reverse, via the LHE technique one constructs the 3D density in a single step, imposing the full 3D cubic symmetry of the momentum density. In the case of $\mathrm{ErGa}_{3}$, the algorithm (labelled as $\mathrm{LHE}(6)$ ) reproduced with good accuracy six density components.

Figure 2 shows that the FBP and MFBP-1 techniques produce strong fluctuations, due to a too large angular step $\Delta \varphi$. When $\Delta \varphi=1^{\circ}, \mathrm{MFBP}$ method gives similar results to CM and LHE algorithms apart from the electron pocket around the $\mathrm{X}$ point which is somehow enhanced by LHE. Let us note that whereas the $\mathrm{CM}$ and FBP results have been symmetrized to impose the 3D cubic symmetry (as described below), the LHE results have the proper 3D symmetry by construction. The symmetrization of the CM and FBP data is accomplished through the following conditions:

$$
\rho\left(p_{x}, p_{y}, p_{z}\right)=\rho\left(p_{y}, p_{z}, p_{x}\right)=\rho\left(p_{x}, p_{z}, p_{y}\right) .
$$

The constraints reconcile densities with the requirement that the nonequiv- 


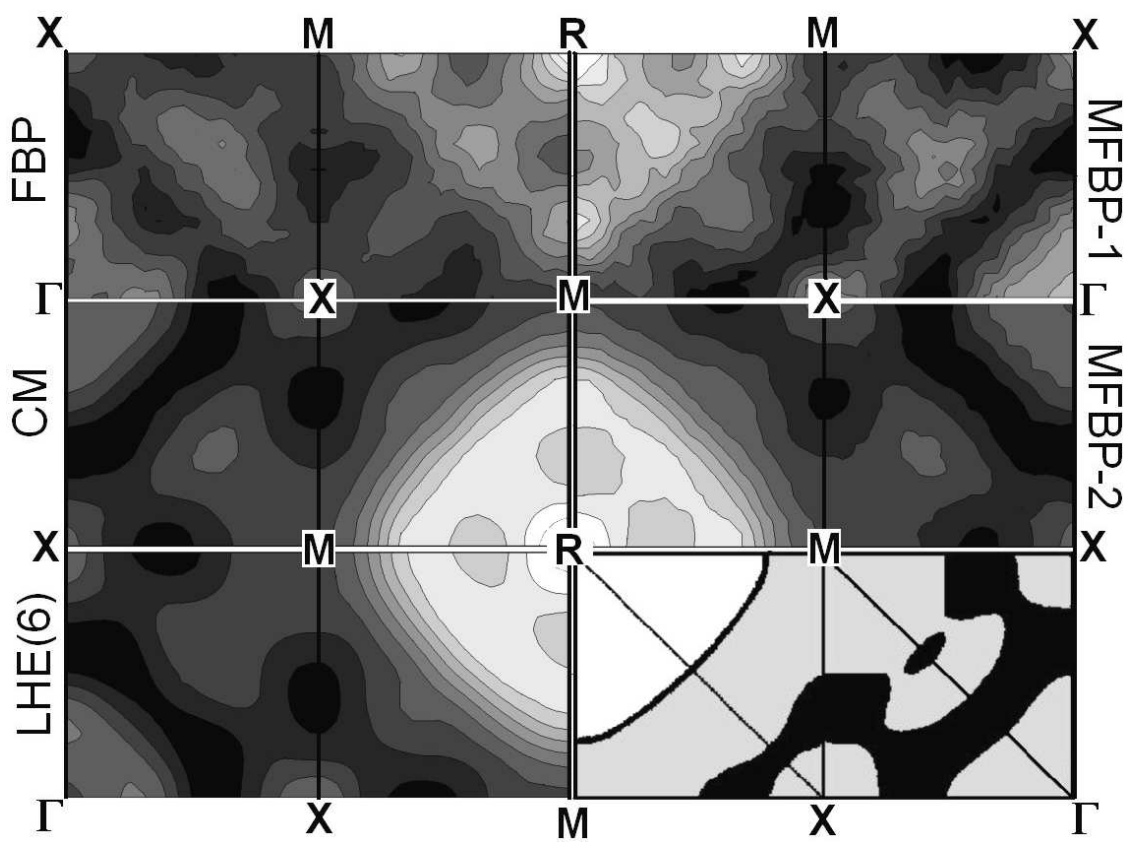

Fig. 2. Densities $\rho(\boldsymbol{p})$ in $\mathrm{ErGa}_{3}$, on high symmetry planes, reconstructed by FBP, MFBP-1, MFBP-2, CM and LHE(6) techniques, compared with the Fermi surface sections calculated via linear muffin-tin orbital-atomic spheres approximation (LMTO-ASA) band structure code [18]. The white regions centered at the $R$ point contain occupied (electrons) from the 7 th valence band and the darkest areas correspond to unoccupied states (holes) from the 6th band.

alent fraction of the Brillouin zone (BZ) is equal to $1 / 48$, unlike the requirements of the structures with one 4 -fold main rotation axis $\left(C_{4 v}\right.$ group), limited to the equivalency of 1/16 of BZ. As explained in detail in Ref. [17] the symmetrization operated with Eq. (7) is similar, but not the same, to the steps of the LHE filtering algorithm.

Figure 3 shows the anisotropic part of the densities in the extended zone of the simple cubic compound $\mathrm{CeIn}_{3}$ reconstructed from five or three 2D ACAR spectra [19]. The 2D densities were reconstructed by CM independently of each other (and for independently measured data set) on planes perpendicular to the [001] direction. Figure 3 shows 60 planes with spacing 0.02 (a.u.) ${ }^{-1}$ (atomic units of momentum).

What is the difference between the CM and LHE reconstruction techniques? Since the densities on the planes (001) are described by the cosine Fourier series (Eq. (2)) with $n=0,4, \ldots$ etc., one has the correct symmetry with regard to the [110] direction. Depending on the number of experimental projections, in Eq. (3) we can use $3[n=0,4,8, \mathrm{CM}(3)]$ or 5 terms $[n=0,4,8,12,15, \mathrm{CM}(5)]$. 


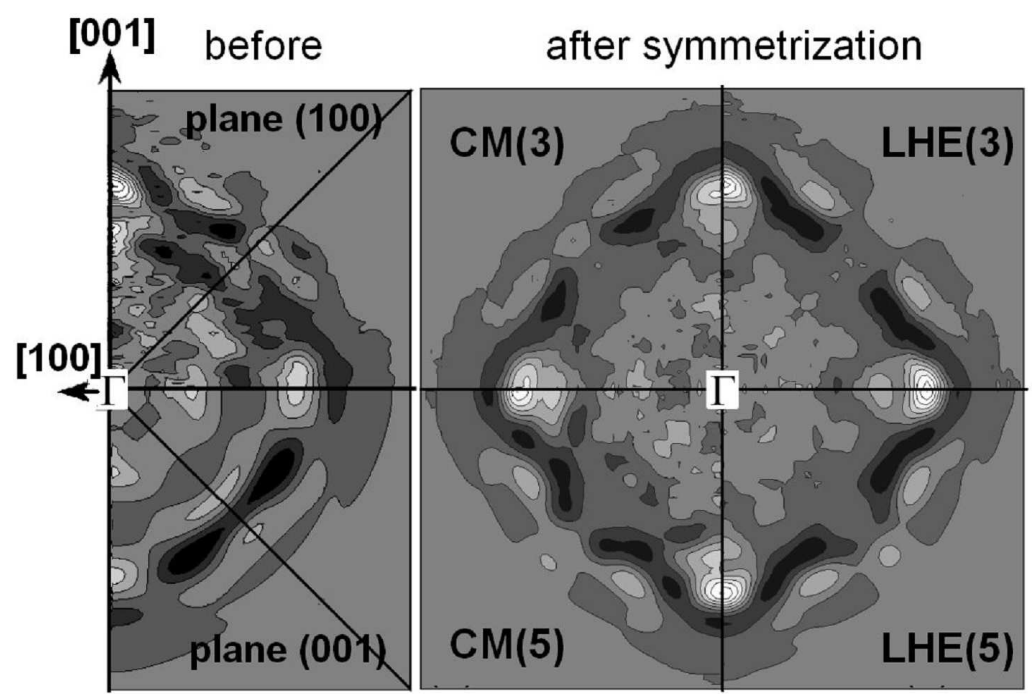

Fig. 3. The anisotropic part of densities reconstructed from three or five projections in $\mathrm{CeIn}_{3}$ by using two reconstruction algorithms (CM and LHE) in the momentum range $(0,1.2) \times(0,1.2)(\text { a.u. })^{-2}$. Left part - results on two planes perpendicular to each other for $\mathrm{CM}(3)$, i.e the $\mathrm{CM}$ applied to 3 projections. Right part — reconstructed densities after symmetrization.

Results for $\mathrm{CM}(3)$ show that three terms $\cos (n \varphi)$ cannot describe densities properly (even on the planes (001) where they are too much smeared). The density on the (100) plane shows clearly undesirable effects: (i) lack of symmetry with regard to [101] axis, (ii) large discrepancies between the two equivalent planes (001) and (100).

However, after imposing on densities the symmetry requirement [Eq. (7)], the results for CM are noticeably improved and become very similar to LHE results. It is worth noting that the LHE method enhances the anisotropy and the results for $\operatorname{LHE}(3)$ are very similar to those produced via $\mathrm{LHE}(5)$. Meanwhile, results for $\mathrm{CM}(3)$ are somewhat different, e.g. there are lower amplitude variation of the maxima along the [100] direction.

\section{Conclusions}

In summary, we can draw the following conclusions:

1. After a simple modification, the FBP method, which is very simple to use, yields for projections without statistical noise the same results as CM.

2. However, since the CM is based on expansions of experimental data into orthogonal polynomials, essentially it reduces the experimental noise when applied to real data. The expansion has a similar effect as a mean squares fitting procedure.

3. Compared to CM (even with symmetry conditions (7)), the LHE algorithm, due to its stricter symmetry requirements [17], filters better the exper- 
imental noise and enhances fine features of the reconstructed densities. These advantages are pronounced when LHE is applied to densities reconstructed from a small number of projections (e.g. for three projections, as shown for $\mathrm{CeIn}_{3}$ here and for $\mathrm{LaB}_{6}$ in Ref. [10]).

4. Our experience points out that (i) it is sufficient to measure 5 projections in the case of cubic structures; (ii) assuming a total number of counts $C$ from all the measured projections it is preferable to concentrate the counts into fewer high-statistics experimental data sets instead of several low-statistics spectra. After the data taking, a standard reconstruction technique such as the Cormack method, together with the symmetry requirement, should produce acceptable results.

\section{References}

[1] S.R. Deans, The Radon Transform and Some of Its Applications, Wiley, New York 1983.

[2] F. Natterer, The Mathematics of Computerized Tomography, Wiley, Stuttgart, 1986.

[3] S. Berko, in: Proc. Int. School Phys. Enrico Fermi Course LXXXIII, Eds. W. Brandt, A. Dupasquier, North Holland, Amsterdam 1983, p. 64.

[4] A.M. Cormack, J. Appl. Phys. 35, 2908 (1964).

[5] G. Kontrym-Sznajd, Phys. Status Solidi A 117, 227 (1990).

[6] A. Brooks, G. Di Chiro, Phys. Med. Biol. 21, 689 (1976).

[7] G. Kontrym-Sznajd, E. Józefczuk, Mater. Sci. Forum 255-257, 754 (1997).

[8] R.N. Brackwell, The Fourier Transform and Its Applications, McGraw Hill, New York 1965, p. 191.

[9] M. Biasini, G. Ferro, G. Kontrym-Sznajd, A. Czopnik, Phys. Rev. B 70, 125103 (2004)

[10] G. Kontrym-Sznajd, M. Samsel-Czekała, M. Biasini, Y. Kubo, Phys. Rev. B 70, 125103 (2004).

[11] H. Matsui, T. Goto, S. Kunii, S. Sakatsume, Physica B 186-188, 126 (1993).

[12] T. Suzuki, T. Goto, T. Fujimura, S. Kunii, T. Suzuki, T. Kasuya, J. Magn. Magn. Mater. 52, (1985); T. Suzuki, T. Goto, Y. Ohe, T. Fujimura, S. Kunii, J. Phys. C 8, 799 (1988).

[13] M. Biasini, H.M. Fretwell, S.B. Dugdale, M.A. Alam, Y. Kubo, H. Harima, N. Sato, Phys. Rev. B 56, 10192 (1997); M. Biasini, M.A. Monge, G. Kontrym-Sznajd, M. Gemmi, N. Sato, Mater. Sci. Forum 363-365, 582 (2001).

[14] F.M. Hossain, D.P. Riley, G.E. Murch, Phys. Rev. B 70, 235101 (2005).

[15] H. Harima, O. Sakai, T. Kasuya, A. Yanase, Solid State Commun. 66, 603 (1988).

[16] Y. Kubo, S. Asano, Phys. Rev. B 39, 8822 (1989).

[17] G. Kontrym-Sznajd, M. Samsel-Czekała, Appl. Phys. A 89, 975 (2007).

[18] V.B. Pluznikov, A. Czopnik, G.E. Grechnev, J. Phys., Condens. Matter 11, 4507 (1999).

[19] M. Biasini, G. Ferro, A. Czopnik, Phys. Rev. B 68, 094513 (2003); J. Rusz, M. Biasini, Phys. Rev. B 71, 233103 (2005). 